\title{
The Spatial Stiffness Matrix from Simple Stretched Springs
}

\author{
J.M. Selig \\ School of Computing, Info. Sys. \& Maths. \\ South Bank University \\ London SE1 0AA, U.K.
}

\begin{abstract}
This work looks at the stiffness matrix of some simple but very general systems of springs supporting a rigid body. The stiffness matrix is found by symbolically differentiating the potential function. After a short example attention turns to the general structure of the stiffness matrix and in particular the principal screws introduced by Ball.
\end{abstract}

\section{Introduction}

There has been much previous work in the area of compliance and stiffness in robots. Most of the work has taken a straightforward approach to the subject, postulating a stiffness or compliance matrix and often a centre of compliance and compliance frame.

A more sophisticated approach was initiated by Lončarić [5] who studied simple systems, a mass supported by a number of different springs. Lončarić discussed the potential energy function of the system but does not seem to have used it to compute the stiffness matrices which are the main subject of his work.

Later, Huang and Schimmels [4], pointed out that not all stiffness matrices could be formed from the simple spring systems studied by Lončarić. They found a simple condition on the trace of part of the stiffness matrix which, if satisfied, guarantees that the matrix can be realised by a system of simple springs. As part of the proof an algorithm was given to find a system of springs which realise the matrix. Most recently Roberts [7] has sharpened this algorithm to find the minimal number of springs needed.

In all of this work the models used for the springs are very simple, they obey Hooke's law and are always unstretched. That is the distance between the endpoints of the spring is always the natural length of the spring. Hence the position studied is always an equilibrium position of the system. Griffis and Duffy [3] noted that, away from equilibrium configurations the stiffness matrix can be unsymmetrical. This is not too surprising since the stiffness matrix is only a symmetric tensor at equilibrium configurations. In the classical mechanics literature it is not usually defined for other positions.

Žefran and Kumar [9], showed how to construct a symmetric tensor field which restricts to the stiffness matrix at an equilibrium point. However, the physical significance of this tensor field is far from clear, especially as it depends on a choice of a connection for the group of rigid body motions.

In this paper the potential function for a simple systems of stretched springs is studied. The resulting wrench is derived by differentiating the potential. This also gives conditions for equilibrium of the system. Differentiating again we obtain the stiffness matrix, so long as the position is an equilibrium.

There are several advantages of using stretched springs. In particular there is a greater flexibility for the designer. When using stretched springs, if the deflections are not too large we can use 'one-sided' springs which can only pull, say.

To first order, an unstretched spring can only produce forces along its length, stretched springs can produce forces perpendicular to their length. This effect is used in trampolines, for example, where the stretched springs are parallel to the bed but the main forced produced is perpendicular to the bed.

The analysis for stretched springs can be reduced, very simply, to the case of unstretched springs.

\section{Differentiation along a screw}

We need to be able to find the gradient of a potential function on a manifold and also the derivative of a vector field. We do this by differentiating along vector fields. In particular, since the manifold is a Lie group, we use elements of the Lie algebra thought of a 
left invariant vector fields. To differentiate using the vector field we move the function a little way along a path tangent to the vector field and look at the limit of the difference between values of the function at neighbouring points.

Let:

$$
S=\left(\begin{array}{cc}
\Omega & \mathbf{v} \\
0 & 0
\end{array}\right)
$$

be a Lie algebra element or screw given in the $4 \times 4$ representation. The $3 \times 3$ anti-symmetric matrix $\Omega=$ $\operatorname{ad}(\boldsymbol{\omega})$, corresponds to a vector $\boldsymbol{\omega}$. So that, $\Omega \mathbf{x}=\boldsymbol{\omega} \times \mathbf{x}$. The notation $\operatorname{ad}()$ refers to the adjoint representation of the rotation group.

If $M$ is a group element written in the $4 \times 4$ representation, then the action of $S$ on $M$, is given by the left translation,

$$
M(t)=e^{t S} M
$$

This takes $M$ along a path tangent to the vector field defined by $S$. Taking the derivative along the path and then setting $t=0$ gives,

$$
\partial_{S} M=S M
$$

\section{$3 \quad$ Springs}

Consider a rigid body supported by a system of springs, assume that the springs obey Hook's law and can both push and pull. The spring constant of the $i$-th spring will be $\lambda_{i}$ and its natural length $l_{i}$. Let $\tilde{\mathbf{a}}_{i}^{T}=\left(\mathbf{a}_{i}^{T}, 1\right)$ be the points where the springs are attached to the ground or frame, and $\tilde{\mathbf{b}}_{i}^{T}=\left(\mathbf{b}_{i}^{T}, 1\right)$ the corresponding attachment points on the rigid body when the body is in some standard 'home' configuration. If the body undergoes a rigid motion the attachment points will move to,

$$
\left(\begin{array}{c}
\mathbf{b}_{i}^{\prime} \\
1
\end{array}\right)=\left(\begin{array}{cc}
R & \mathbf{t} \\
0 & 1
\end{array}\right)\left(\begin{array}{c}
\mathbf{b}_{i} \\
1
\end{array}\right)
$$

which we will abbreviate to $\tilde{\mathbf{b}}_{i}^{\prime}=M \tilde{\mathbf{b}}_{i}$.

Let us write,

$$
\mathbf{p}_{i}=\left(R \mathbf{b}_{i}+\mathbf{t}-\mathbf{a}_{i}\right) \quad \text { and } \quad \hat{\mathbf{p}}_{i}=\mathbf{p}_{i} /\left|\mathbf{p}_{i}\right|
$$

for the vector and unit vector along the $i$ th spring. We can then write the extension of the $i$ th spring as, $\mathbf{e}_{i}=\mathbf{p}_{i}-l_{i} \hat{\mathbf{p}}_{i}$.

Now we can write the potential energy of the system as,

$$
\Phi=\frac{1}{2} \sum_{i} \lambda_{i} \mathbf{e}_{i}^{T} \mathbf{e}_{i}
$$

Notice that this function is defined on the group of rigid motions, as $R$ and $\mathbf{t}$ change the potential will vary.

To find the wrench due to the springs we differentiate the potential. In general a wrench is a 6dimensional vector of forces and torques,

$$
\mathcal{W}=\left(\begin{array}{l}
\boldsymbol{\tau} \\
\mathbf{F}
\end{array}\right)
$$

where $\boldsymbol{\tau}$ is a moment about the origin and $\mathbf{F}$ is a force. Notice that wrenches are not Lie algebra elements but elements of the vector space dual to the Lie algebra. Usually the force due to a potential is given by its gradient. The same is true here, in terms of the exterior derivative $d$ we have, $\mathcal{W}=-d \Phi$. Pairing the wrench with an arbitrary screw $S$ gives,

$$
\mathcal{W}(S)=-d \Phi(S)=-\partial_{S} \Phi
$$

see $[8, \S .4 .20]$ for example.

To find the derivative of the potential with respect to an arbitrary screw $S$ we first look at the derivative of the unit vector $\hat{\mathbf{p}}_{i}=\left(\mathbf{p}_{i}^{T} \mathbf{p}_{i}\right)^{-1 / 2} \mathbf{p}_{i}$. We obtain the result,

$$
\partial_{S} \hat{\mathbf{p}}_{i}=\frac{1}{\left|\mathbf{p}_{i}\right|^{3}}\left(\left(\mathbf{p}_{i}^{T} \mathbf{p}_{i}\right) I_{3}-\left(\mathbf{p}_{i} \mathbf{p}_{i}^{T}\right)\right) \partial_{S} \mathbf{p}_{i}
$$

The derivative of the potential energy is thus,

$$
\partial_{S} \Phi=\sum_{i} \lambda_{i} \mathbf{e}_{i}^{T}\left(I_{3}-\frac{l_{i}}{\left|\mathbf{p}_{i}\right|} I_{3}+\frac{l_{i}}{\left|\mathbf{p}_{i}\right|^{3}}\left(\mathbf{p}_{i} \mathbf{p}_{i}^{T}\right)\right) \partial_{S} \mathbf{p}_{i}
$$

Evaluating the derivative at the home position gives,

$$
\partial_{S} \mathbf{p}_{i}=\left(\begin{array}{ll}
B_{i}^{T} & I_{3}
\end{array}\right)\left(\begin{array}{c}
\boldsymbol{\omega} \\
\mathbf{v}
\end{array}\right)
$$

After simplification we obtain,

$$
\mathcal{W}=-\left(\begin{array}{c}
\sum_{i} \lambda_{i} \mathbf{b}_{i} \times \mathbf{e}_{i} \\
\sum_{i} \lambda_{i} \mathbf{e}_{i}
\end{array}\right)
$$

which could also have been deduced from elementary mechanics. The point is that these methods do indeed agree with more fundamental methods.

In the following it will be more convenient to use the equivalent formula for the wrench,

$$
\mathcal{W}=-\left(\begin{array}{c}
\sum_{i} \lambda_{i} \mathbf{a}_{i} \times \mathbf{e}_{i} \\
\sum_{i} \lambda_{i} \mathbf{e}_{i}
\end{array}\right)
$$

since the $\mathbf{a}_{i}$ s are fixed. Equilibrium positions are determined by the vanishing of this wrench. The resulting pair of vector equations are very difficult to solve symbolically.

Problems concerning the existence of solutions do not arise when using unstretched springs. If all the springs are unstretched in the home position then $\mathbf{e}_{i}=$ 0 for all $i$ and so home is an equilibrium position. 
Figure 1: A Rigid Body Suspended by a Pair of Springs

\section{The Stiffness Matrix}

Here, we derive the stiffness matrix from the potential given above. That is, we look at the linearisation of the the system of springs. An infinitesimal displacement of the body is represented by a screw. Here we prefer the six-dimensional representation of screw, $\mathbf{s}^{T}=\left(\boldsymbol{\omega}^{T}, \mathbf{v}^{T}\right)$, where $\boldsymbol{\omega}$ is an angular displacement and $\mathbf{v}$ a linear one. The wrench produced by a displacement $\mathbf{s}$ is given by $\mathcal{W}=K \mathbf{s}$, where $K$ is the stiffness matrix.

The stiffness matrix is the hessian of the potential function, that is its matrix of second order partial derivatives, see $[1, \mathrm{Ch} 5]$. This is only valid at an equilibrium position.

Above we saw that,

$$
\partial_{S} \Phi=\left(\begin{array}{c}
\sum_{i} \lambda_{i} \mathbf{a}_{i} \times \mathbf{e}_{i} \\
\sum_{i} \lambda_{i} \mathbf{e}_{i}
\end{array}\right)^{T}\left(\begin{array}{c}
\boldsymbol{\omega} \\
\mathbf{v}
\end{array}\right)
$$

To find the second derivative we only need to know $\partial_{S} \mathbf{e}_{i}$ which we have already derived. If we write,

$$
X_{i}=\frac{1}{\left|\mathbf{p}_{i}\right|}\left(\left(\left|\mathbf{p}_{i}\right|-l_{i}\right) I_{3}+l_{i}\left(\hat{\mathbf{p}}_{i} \hat{\mathbf{p}}_{i}^{T}\right)\right)
$$

Then the stiffness matrix, $K=\left(\begin{array}{cc}\Xi & \Gamma \\ \widetilde{\Gamma}^{T} & \Upsilon\end{array}\right)$ can be written as,

$$
\begin{aligned}
& \Xi=\sum_{i} \lambda_{i} A_{i} X_{i} B_{i}^{T} \quad \Gamma=\sum_{i} \lambda_{i} A_{i} X_{i} \\
& \widetilde{\Gamma}^{T}=\sum_{i} \lambda_{i} X_{i} B_{i}^{T} \quad \Upsilon=\sum_{i} \lambda_{i} X_{i}
\end{aligned}
$$

The apparent asymmetry here is accounted for by the fact that we have not imposed the condition that the position of the body is an equilibrium configuration. This is a necessary condition for the stiffness matrix to be a symmetric matrix and hence to be physically meaningful. To see this notice that in the home position $\mathbf{p}_{i}=\mathbf{b}_{i}-\mathbf{a}_{i}$ and so we can write,

$$
\begin{aligned}
\Xi=\sum_{i}\left(\frac{\lambda_{i}}{\left|\mathbf{p}_{i}\right|}\left(\left|\mathbf{p}_{i}\right|-l_{i}\right) A_{i} B_{i}^{T}+\right. & \\
& \left.\frac{\lambda_{i} l_{i}}{\left|\mathbf{p}_{i}\right|^{3}}\left(\mathbf{a}_{i} \times \mathbf{b}_{i}\right)\left(\mathbf{a}_{i} \times \mathbf{b}_{i}\right)^{T}\right)
\end{aligned}
$$

Hence if we take the difference between $\Xi$ and its transpose we get,

$$
\begin{aligned}
\Xi^{T}-\Xi & =\sum_{i} \frac{\lambda_{i}}{\left|\mathbf{p}_{i}\right|}\left(\left|\mathbf{p}_{i}\right|-l_{i}\right)\left(B_{i} A_{i}^{T}-A_{i} B_{i}^{T}\right) \\
& =\sum_{i} \lambda_{i} \operatorname{ad}\left(\mathbf{a}_{i} \times \mathbf{e}_{i}\right)=0
\end{aligned}
$$

when the home position is an equilibrium position. This shows that $\Xi$ is symmetric, $\Upsilon$ is clearly symmetric and a similar argument shows that $\widetilde{\Gamma}=\Gamma$.

Notice that if we set $\left|\mathbf{p}_{i}\right|=l_{i}$ we recover the stiffness matrix for unstretched springs.

\section{An Example}

To illustrate the above theory we look at a very simple system consisting of a symmetrical pair of springs, see fig. 1. We sidestep the difficulties in finding an equilibrium position, the symmetry of the setup makes it clear that the position shown is an equilibrium.

Using $\mathbf{i}$ for the unit vector in the $x$-direction, we have, $\mathbf{p}_{R}=-(l+e) \mathbf{i},\left|\mathbf{p}_{R}\right|=l+e$ and on the left side $\mathbf{p}_{L}=(l+e) \mathbf{i},\left|\mathbf{p}_{L}\right|=l+e$. From this we can calculate,

$$
X_{R}=X_{L}=\left(\begin{array}{ccc}
1 & 0 & 0 \\
0 & \frac{e}{l+e} & 0 \\
0 & 0 & \frac{e}{l+e}
\end{array}\right)
$$

Now since,

$$
A_{R}=-A_{L}=\left(\begin{array}{ccc}
0 & 0 & 0 \\
0 & 0 & -(1+l+e) \\
0 & (1+l+e) & 0
\end{array}\right)
$$

we get that $A_{R} X_{R}=-A_{L} X_{L}$ where,

$$
A_{R} X_{R}=\left(\begin{array}{ccc}
0 & 0 & 0 \\
0 & 0 & -e\left(1+\frac{1}{l+e}\right) \\
0 & e\left(1+\frac{1}{l+e}\right) & 0
\end{array}\right)
$$


and $A_{R} X_{R} B_{R}^{T}=A_{L} X_{L} B_{L}^{T}$ giving,

$$
A_{R} X_{R} B_{R}^{T}=\left(\begin{array}{ccc}
0 & 0 & 0 \\
0 & e\left(1+\frac{1}{l+e}\right) & 0 \\
0 & 0 & e\left(1+\frac{1}{l+e}\right)
\end{array}\right)
$$

So for this very simple system the stiffness matrix has blocks,

$$
\begin{aligned}
\Xi & =\left(\begin{array}{ccc}
0 & 0 & 0 \\
0 & 2 \lambda e\left(1+\frac{1}{l+e}\right) & 0 \\
0 & 0 & 2 \lambda e\left(1+\frac{1}{l+e}\right)
\end{array}\right) \\
\Upsilon & =\left(\begin{array}{ccc}
2 \lambda & 0 & 0 \\
0 & 2 \lambda \frac{e}{l+e} & 0 \\
0 & 0 & 2 \lambda \frac{e}{l+e}
\end{array}\right)
\end{aligned}
$$

and $\Gamma=0$.

This result shows clearly the difference between stretched and unstretched springs. The unstretched case can be found by setting $e=0$. Notice that in the case of stretched springs a deflection in the $y$ direction produces a force in the $y$-direction. A small twist about the $y$ or $z$-axes will also produce a torque about the corresponding axis. But no twist about the $x$-axis, the direction of the springs, will produce any torque.

We can make our simple example a little more general by considering the effect of rigid motion on the springs. Suppose that we subject the springs, that is their attachment points $\mathbf{a}_{i}$ and $\mathbf{b}_{i}$ to a rotation $R$ followed by a translation $T=\operatorname{ad}(\mathbf{t})$. From the relation, $\mathcal{W}=K \mathrm{~s}$ and the transformation properties of screws and wrenches, we can deduce that the effect on the stiffness matrix $K$ is given by,

$$
K^{\prime}=H^{-T} K H^{-1}
$$

where $H=\left(\begin{array}{cc}R & 0 \\ T R & R\end{array}\right)$ is the $6 \times 6$ matrix which transform a screw. In this way we can build-up the stiffness matrix for complicated arrangements of springs from springs aligned with the coordinate axes.

Notice that, after a translation we will have $\Gamma \neq 0$, even if it was zero to begin with.

\section{The Trace Condition}

Huang and Schimmels [4] showed that for unstretched springs the stiffness matrix satisfies the trace condition $^{\dagger}, \operatorname{Tr}\left(K Q_{0}\right)=0$ where, $Q_{0}=\left(\begin{array}{cc}0 & I_{3} \\ I_{3} & 0\end{array}\right)$.

\footnotetext{
†This condition also appears in a footnote in Lončarić [5]
}

The condition is equivalent to $\operatorname{Tr}(\Gamma)=0$ and was a consequence of the fact that for unstretched springs the contribution to the stiffness matrix from the $i$-th spring is proportional to $\mathcal{W}_{i} \mathcal{W}_{i}^{T}$, where $\mathcal{W}_{i}$ is a pure force directed along the $i$-th spring. This is not the case for stretched springs as we have seen above, however, the trace condition still holds since,

$$
\begin{aligned}
\operatorname{Tr}(\Gamma)=\sum_{i}\left(\frac{\lambda_{i}}{\left|\mathbf{p}_{i}\right|}\left(\left|\mathbf{p}_{i}\right|-l_{i}\right) \operatorname{Tr}\left(A_{i}\right)+\right. \\
\left.\frac{\lambda_{i} l_{i}}{\left|\mathbf{p}_{i}\right|^{3}} \operatorname{Tr}\left(\left(\mathbf{a}_{i} \times \mathbf{p}_{i}\right) \mathbf{p}_{i}^{T}\right)\right)=0
\end{aligned}
$$

This vanishes because $A_{i}$ is antisymmetric, and by the cyclic properties of the trace operator, $\operatorname{Tr}\left(\left(\mathbf{a}_{i} \times\right.\right.$ $\left.\left.\mathbf{p}_{i}\right) \mathbf{p}_{i}^{T}\right)=\mathbf{p}_{i} \cdot \mathbf{a}_{i} \times \mathbf{p}_{i}=0$.

The condition is invariant with respect to rigid transformations. A simple computation shows that $H Q_{0} H^{T}=Q_{0}$ and so,

$$
\operatorname{Tr}\left(K^{\prime} Q_{0}\right)=\operatorname{Tr}\left(H^{-T} K H^{-1} H Q_{0} H^{T}\right)=\operatorname{Tr}\left(K Q_{0}\right)
$$

The matrix $Q_{\infty}=\left(\begin{array}{cc}0 & 0 \\ 0 & I_{3}\end{array}\right)$ also satisfies $H Q_{\infty} H^{T}=Q_{\infty}$ and hence $\operatorname{Tr}\left(K Q_{\infty}\right)$ is another invariant. This invariant is equivalent to $\operatorname{Tr}(\Upsilon)$ and was also found by Lončarić [5]. For our stretched springs we get,

$$
\operatorname{Tr}(\Upsilon)=\sum_{i} \lambda_{i} \operatorname{Tr}\left(X_{i}\right)=\sum_{i} \lambda_{i}\left(3-2 \frac{l_{i}}{\left|\mathbf{p}_{i}\right|}\right)
$$

\section{Normal Forms}

A rigid change of coordinates will transform the stiffness matrix according to,

$$
K^{\prime \prime}=H^{T} K H
$$

this is the inverse of the active transformation we met in section 5 . In terms of $3 \times 3$ submatrices the transformation can be written as,

$$
\begin{aligned}
\Xi^{\prime \prime} & =R^{T} \Xi R+R^{T} \Gamma T R-R^{T} \Gamma^{T}-R^{T} T \Upsilon T R \\
\Gamma^{\prime \prime} & =R^{T} \Gamma R-R^{T} T \Upsilon R \\
\Upsilon^{\prime \prime} & =R^{T} \Upsilon R
\end{aligned}
$$

In [5] Lončarić showed that it is always possible to translate the coordinate frame to a position where $\Gamma$ is symmetric, provided $\operatorname{det}\left(\Upsilon-\operatorname{Tr}(\Upsilon) I_{3}\right) \neq 0$. Then a rotation can be found which will diagonalise $\Gamma$. So in Lončarić's normal form $\Xi$ and $\Upsilon$ are symmetric and $\Gamma$ 
is diagonal (and traceless for stretched springs). However, the final rotation could have been chosen to diagonalise $\Xi$ or $\Upsilon$. There are also alternatives to symmetrising $\Gamma$, so there are many different possible normal forms for the stiffness matrix.

The advantage of the Lončarić normal form, according to [5], is that the frame "maximally decouples rotational and translational aspects of stiffness." To understand what this might mean we look at the existence of centres of compliance and compliance frames. These ideas are much used in practical work but there does not seem to be a great deal work on the conditions for the existence of centres of compliance. In the literature there are several different definitions of the centre of compliance.

One definition of the centre of compliance is, a point at which a pure force produces a pure translation in the same direction. If we write the centre as the point c then have the following equation,

$$
\left(\begin{array}{cc}
\Xi & \Gamma \\
\Gamma^{T} & \Upsilon
\end{array}\right)\left(\begin{array}{l}
\mathbf{0} \\
\mathbf{v}
\end{array}\right)=\beta\left(\begin{array}{c}
\mathbf{c} \times \mathbf{v} \\
\mathbf{v}
\end{array}\right)
$$

The equations is required to hold for any vector $\mathbf{v}$, the constant $\beta$ is arbitrary. The relation $\Gamma \mathbf{v}=\beta \mathbf{c} \times \mathbf{v}$ tells us that, $\Gamma=\beta \operatorname{ad}(\mathbf{c})=\beta C$ an anti-symmetric matrix. The second relation, $\Upsilon \mathbf{v}=\beta \mathbf{v}$ means that $\Upsilon=\beta I_{3}$, this is $\Upsilon$ is proportional to the identity matrix. Now translating by $\mathbf{c}$ we can make $\Gamma$ symmetric. In fact, in this coordinate frame $\Gamma=0$. This coordinate frame is clearly the Lončaric frame and the centre of compliance is the origin in this frame.

We can do the same with torques and rotations, if the compliance centre is defined as the point where and pure torque results in a pure rotation about the same axis we obtain the equation,

$$
\left(\begin{array}{cc}
\Xi & \Gamma \\
\Gamma^{T} & \Upsilon
\end{array}\right)\left(\begin{array}{c}
\boldsymbol{\omega} \\
\mathbf{c} \times \boldsymbol{\omega}
\end{array}\right)=\alpha\left(\begin{array}{l}
\boldsymbol{\omega} \\
\mathbf{0}
\end{array}\right)
$$

In the Lončarić frame we have $\Gamma=0$ and $\Xi=\alpha I_{3}$. So the rotation axes will all pass through the origin of the Lončarić frame.

Putting these definitions for the compliance centre together we get the definition given in $[6, \S 6.1 .2]$ for example, a point at which a pure force produces a pure translation in the same direction and pure torque results in a pure rotation about the same axis. Now it is not hard to see that the conditions for such a point to exist are that the stiffness matrix must have the Lončarić normal form,

$$
K=\left(\begin{array}{cc}
\alpha I_{3} & 0 \\
0 & \beta I_{3}
\end{array}\right)
$$

where $\alpha$ and $\beta$ are constants. Moreover, the position of the centre of compliance is the origin of the coordinate frame. In more general coordinates, this stiffness matrix has the same shape as the inertia matrix of a spherically symmetric object.

Notice that it is not too difficult to design such a stiffness matrix using stretched springs. One possibility would be to use pairs of identical springs stretched so that their lines of action all pass through a common point. (We must necessarily have $\beta=\operatorname{Tr}(\Upsilon) / 3$.)

\section{Ball's Principal Screws}

In his famous treatise [2] Ball described what he called principal screws of potential. A principal screw is a displacement which produces a wrench with the same pitch and axis as the screw. They are solutions to the following eigenproblem,

$$
K \mathbf{s}=\mu Q_{0} \mathbf{s}
$$

In general we get six eigenvalues $\mu$, the solutions to the characteristic equation,

$$
\operatorname{det}\left(K-\mu Q_{0}\right)=0
$$

and a principal screw or eigenscrew s, corresponding to each eigenvalue. This is very similar to the standard eigenvalue problem and many of the standard theorems apply directly or can be modified slightly for the present situation. For example, if the eigenvalues are all different and non-zero we have that a pair of different eigenscrews $\mathbf{s}_{1}$ and $\mathbf{s}_{2}$ will satisfy,

$$
\mathbf{s}_{1}^{T} Q_{0} \mathbf{s}_{2}=0 \quad \text { and } \quad \mathbf{s}_{1}^{T} K \mathbf{s}_{2}=0
$$

In the first relation we say the screws are reciprocal, this is the analogue of the eigenvectors being orthogonal. Ball called screws obeying the second relation, conjugate screws of potential.

From this we can say something about the principle screws. For example, is it possible for an eigenscrew to be a pure translation? The equation,

$$
\left(\begin{array}{cc}
\Xi & \Gamma \\
\Gamma^{T} & \Upsilon
\end{array}\right)\left(\begin{array}{l}
\mathbf{0} \\
\mathbf{v}
\end{array}\right)=\mu\left(\begin{array}{l}
\mathbf{v} \\
\mathbf{0}
\end{array}\right)
$$

shows that this can only happen if $\Upsilon$ is singular. A similar argument shows that a principal screw can only be a pure rotation if $\Xi$ is singular.

Is it possible to have two different principal screws with the same direction? Let two such screws be,

$$
\mathbf{s}_{1}=\left(\begin{array}{c}
\boldsymbol{\omega} \\
\mathbf{r}_{1} \times \boldsymbol{\omega}+\pi_{1} \boldsymbol{\omega}
\end{array}\right), \quad \mathbf{s}_{2}=\left(\begin{array}{c}
\boldsymbol{\omega} \\
\mathbf{r}_{2} \times \boldsymbol{\omega}+\pi_{2} \boldsymbol{\omega}
\end{array}\right)
$$


The relation $\mathbf{s}_{1}^{T} Q_{0} \mathbf{s}_{2}=0$ reduces to $\left(\pi_{1}+\pi_{2}\right) \boldsymbol{\omega} \cdot \boldsymbol{\omega}=$ 0 . Hence this can only happen if the pitches of the principal screws are equal and opposite.

Can two principal screws have axes which pass through the same point? Consider the screws,

$$
\mathbf{s}_{1}=\left(\begin{array}{c}
\boldsymbol{\omega}_{1} \\
\mathbf{r} \times \boldsymbol{\omega}_{1}+\pi_{1} \boldsymbol{\omega}_{1}
\end{array}\right), \quad \mathbf{s}_{2}=\left(\begin{array}{c}
\boldsymbol{\omega}_{2} \\
\mathbf{r} \times \boldsymbol{\omega}_{2}+\pi_{2} \boldsymbol{\omega}_{2}
\end{array}\right)
$$

Now the fact that the screws must be reciprocal becomes, $\left(\pi_{1}+\pi_{2}\right) \boldsymbol{\omega}_{1} \cdot \boldsymbol{\omega}_{2}=0$. So two principal screw which pass through the same point are either parallel with opposite pitches or orthogonal.

Another standard result which we can modify is the expansion of the matrix in terms of its eigenvectors. It is not hard to see that if a stiffness matrix $K$ has eigenvalues $\mu_{i}$ and corresponding eigenscrews $\mathbf{s}_{i}$ then we can expand the matrix as,

$$
K=\sum_{i=1}^{6} \frac{\mu_{i}}{2 \boldsymbol{\omega}_{i} \cdot \mathbf{v}_{i}} Q_{0} \mathbf{s}_{i} \mathbf{s}_{i}^{T} Q_{0}
$$

For the partitioned form of the matrix we can write,

$$
\left(\begin{array}{cc}
\Xi & \Gamma \\
\Gamma^{T} & \Upsilon
\end{array}\right)=\sum_{i=1}^{6} \frac{\mu_{i}}{2 \boldsymbol{\omega}_{i} \cdot \mathbf{v}_{i}}\left(\begin{array}{cc}
\mathbf{v}_{i} \mathbf{v}_{i}^{T} & \mathbf{v}_{i} \boldsymbol{\omega}_{i}^{T} \\
\boldsymbol{\omega}_{i} \mathbf{v}_{i}^{T} & \boldsymbol{\omega}_{i} \boldsymbol{\omega}_{i}^{T}
\end{array}\right)
$$

From this we can see that the invariants of section 6 can be written in terms of the eigenvalues and the pitches of the eigenscrews $\pi_{i}=\boldsymbol{\omega}_{i} \cdot \mathbf{v}_{i} / \boldsymbol{\omega}_{i} \cdot \boldsymbol{\omega}_{i}$,

$$
\operatorname{Tr}(\Gamma)=\frac{1}{2} \sum_{i=1}^{6} \mu_{i} \quad \text { and } \quad \operatorname{Tr}(\Upsilon)=\frac{1}{2} \sum_{i=1}^{6} \frac{\mu_{i}}{\pi_{i}}
$$

Finally, notice that in the Lončarić normal form, since $\Gamma$ is symmetric we can derive the following relation for the principal screws,

$$
\sum_{i=1}^{6} \frac{\mu_{i}}{\boldsymbol{\omega}_{i} \cdot \mathbf{v}_{i}} \boldsymbol{\omega}_{i} \times \mathbf{v}_{i}=\mathbf{0}
$$

\section{Conclusions}

The principal screws and their eigenvalues are the key structures which characterise the stiffness matrix of a system. The constraint $\operatorname{Tr}(\Gamma)=0$, satisfied by systems of stretched springs, seem quite mild, implying only that the eigenvalues sum to zero.

One reason for looking at systems of stretched springs was to explore simple but physical, models for the desired compliance between a robot and its environment in an active stiffness control scheme. The stretched spring systems cover a wide variety of forms, most of which are not considered in standard control schemes.

A complication introduced by these systems is the problem of finding equilibrium positions. In many cases we can avoid this problem by considering symmetrical designs which have an obvious equilibrium position.

Since stretched springs can produce forces that are not just along their lengths, does this mean that we need fewer stretched springs to produce the same stiffness matrix than could be designed with unstretched springs? This question has not been studied yet.

\section{References}

[1] V.I. Arnol'd. Geometrical Methods of Classical Mechanics, volume 60 of Graduate Texts in Mathematics. Springer-Verlag, New York, 1978.

[2] R.S. Ball. The Theory of Screws. Cambridge University Press, Cambridge, 1900.

[3] M. Griffis and J. Duffy. Global stiffness modelling of a class of simple compliant coupling. Mechanism and Machine Theory, 28(2):207-224, 1993.

[4] S. Huang and J.M. Schimmels. The bounds and realization of spatial stiffnesses achieved with simple springs connected in parallel. IEEE Trans. on Robotics and Automation, 14(3):466-475, 1998.

[5] J. Lončarić. Normal forms of stiffness and compliance matrices. IEEE J. of Robotics and Automation, RA-3(6):567-572, 1987.

[6] P.J. McKerrow. Introduction to Robotics. AddisonWesley, Sydney, 1991.

[7] R.G. Roberts. Minimal realization of spal stiffness matrix with simple springs connected in parallel. In Proceedings of the 1999 IEEE International Conference on Robotics and Automation, Detroit, MI, pages ??-??

[8] B.F. Schutz. Geometrical Methods of Mathematical Physics. Cambridge University Press, Cambridge, 1980.

[9] M. Žefran and V. Kumar. Affine connection for the cartesian stiffness matrix. In Proceedings of the 1997 IEEE International Conference on Robotics and Automation, Albuquerque, NM, pages 13761381. 